\title{
Oxysophoridine attenuates the injury caused by acute myocardial infarction in rats through anti-oxidative, anti-inflammatory and anti-apoptotic pathways
}

\author{
CONG MENG ${ }^{1 *}$, CHUAN LIU ${ }^{2 *}$, YUANWEI LIU ${ }^{1}$ and FAN WU ${ }^{3}$ \\ ${ }^{1}$ Department of Radiology, Huangdao Branch of the Affiliated Hospital of Qingdao University, Qingdao, Shandong 266555; \\ ${ }^{2}$ Department of Clinical Medicine, Yantai Campus, Binzhou Medical University, Yantai, Shandong 264000; \\ ${ }^{3}$ Department of Pediatrics, Dongying Hospital of Shandong Provincial Hospital, Dongying, Shandong 257091, P.R. China
}

Received December 6, 2013; Accepted June 20, 2014

DOI: $10.3892 / \mathrm{mmr} .2014 .2748$

\begin{abstract}
Oxysophoridine (OSR), a natural alkaloid derived from the traditional Chinese medicinal plant sophora alopecuroides, can perform a variety of pharmacological actions. The aim of the present study was to assess the cardioprotective effect of OSR against acute myocardial infarction (AMI) in rats. OSR markedly reduced infarction size and levels of specific myocardial enzymes, including creatine kinase, the MB isoenzyme of creatine kinase, lactate dehydrogenase and cardiac troponin T. A reduced level of malondialdehyde was observed, and elevated catalase, $\mathrm{Cu} / \mathrm{Zn}$-superoxide dismutase (SOD), Mn-SOD, non-enzymatic scavenger glutathione and glutathione peroxidase activity were also identified in the OSR-treated rats. Additionally, OSR inhibited the activities of various inflammatory cytokines in a dose-dependent manner. These included nuclear factor- $\kappa \mathrm{B}$ p 65, tumor necrosis factor- $\alpha$, and interleukin-1 $\beta$, -6 and -10 . Furthermore, OSR treatment suppressed caspase-3 activity in a dose-dependent manner. These results demonstrate that OSR ameliorates cardiac damage in a rat model of AMI and that this cardioprotection may be linked with its anti-oxidative, anti-apoptotic and anti-inflammatory properties.
\end{abstract}

\section{Introduction}

Myocardial infarction is regarded as one of the most common types of ischemic heart disease and a major cause of morbidity and mortality worldwide. It arises from a sudden

Correspondence to: Dr Chuan Liu, Department of Clinical Medicine, Yantai Campus, Binzhou Medical University, 346 Guanhai Road, Yantai, Shandong 264000, P.R. China

E-mail: chuanliudr@163.com

*Contributed equally

Key words: oxysophoridine, acute myocardial infarction, cardioprotection, anti-oxidative, anti-inflammatory, anti-apoptotic and persistent interruption of the myocardial blood supply, leading to a marked loss of cardiomyocytes. Although certain western drugs are used for treating myocardial ischemia, including angiotensin-converting enzyme inhibitors, calcium channel blockers and angiotensin II receptor antagonists, their application is limited by their serious side effects, such as cardiac depression or proarrhythmia (1). Thus, there is an urgent requirement for the development of novel therapeutic approaches to attenuate the damage caused by myocardial infarction.

Substantial evidence has demonstrated that reactive oxygen species (ROS) contribute to cell loss following myocardial infarction. The high level of oxygen free radicals following ischemia was reported to damage cellular structures, cause mitochondrial dysfunction and activate apoptotic signaling cascades (2). In addition, oxidative stress is closely associated with the generation of myocardial ischemia. Thus, the reduction of ROS may serve as an important therapeutic target for attenuating the damage caused by acute myocardial infarction (AMI).

Nuclear factor $(\mathrm{NF})-\kappa \mathrm{B}$ is a vital cellular transcription factor that is present in myocytes. Changes in its expression level have been demonstrated to reflect pathological alterations during AMI $(3,4)$. Activation of $N F-\kappa B$ may trigger the release of proinflammatory cytokines, such as TNF- $\alpha$ and IL-1 $\beta$. In cells undergoing myocardial infarction, high levels of NF- $\kappa \mathrm{B}$ p 65 , TNF- $\alpha$ and IL- $1 \beta$ facilitate myocardial necrosis and cellular apoptosis, subsequently inducing heart failure. Therefore, drugs that inhibit the NF- $\mathrm{B}$ signaling pathway and the release of proinflammatory factors may serve as potential treatments for a variety of cardiovascular disorders.

It is well established that apoptosis participates in the pathogenesis of myocardial injury during myocardial infarction. Caspase-3 is the largest subgroup of the caspase family and acts to induce apoptosis in response to apoptotic stimuli (5). A previous investigation demonstrated a marked elevation in the levels of caspase-3 in response to isoproterenol-induced AMI in Wistar rats (6). These results suggest that modulating caspase-3 may reduce cardiac injury during myocardial infarction. 
A number of traditional Chinese medicines have been indicated to exert a protective role against AMI in animals $(7,8)$. Oxysophoridine (OSR) is a natural alkaloid purified from Sophora alopecuroides $L$. and has been demonstrated to possess diverse pharmacological actions. A previous study by Wang et al (9) demonstrated that OSR was able to perform a protective role against ischemic damage following middle cerebral artery occlusion in mice. They indicated that this neuroprotection may be associated with the suppression of oxidative stress and apoptosis. However, whether OSR can protect against AMI in rats has yet to elucidated. Therefore, the current study was performed to investigate the cardioprotective effects of OSR against AMI in rats and to clarify the underlying molecular mechanisms.

\section{Materials and methods}

Ethical disclosure. All surgical procedures conducted in the present study were approved by the animal ethics committee of the Huangdao Branch of the Affiliated Hospital of Qingdao University Medical College (Qingdao, China).

Animals and AMI production. Adult male Wistar rats (250-300 g) were supplied by the Beijing Laboratory Animal Research Center (Beijing, China). All animals were maintained in individual cages under a standard environment (12/12 h light/dark cycle, 50-70\% humidity, $24^{\circ} \mathrm{C}$ ) and provided with free access to food and water. The induction of rat AMI was performed according to the methods of a previous study (10). Briefly, animals were anesthetized intraperitoneally (i.p.) with $40 \mathrm{mg} / \mathrm{kg}$ sodium pentobarbitone. They were then intubated and artificially ventilated with a respirator (Chengdu Taimeng Technology Co., Chengdu, China). The standard electrocardiogram II (Chengdu Taimeng Technology Co.) was procured by a transducer attached to a multi-channel recorder (BL-420F Data Acquisition \& Analysis System, Chengdu Technology \& Market Co., Ltd., Chengdu, China) following subcutaneous penetration of electrodes into the four limbs. A 5.0 silk suture 1-2 $\mathrm{mm}$ in diameter was used to encircle the left anterior descending coronary artery under the left atrial appendage. Sham-operated animals were treated with the same surgical procedures without the coronary artery ligation. Efforts were made to reduce the number of animals used and minimize their suffering. Successful ligation was verified by the observation of ST-segment elevation and regional cyanosis of the myocardial surface.

Drug administration. Oxysophoridine (OSR; purity, 98\%), supplied by the Institution of Chemistry and Chemical Engineering (Beijing, China) was dissolved in physiological saline. The rats were randomly assigned to five groups as follows: i) The sham-operated group, which was injected with physiological saline $(0.1 \mathrm{ml} / 100$ g, i.p. $)$ and underwent the surgery without the coronary artery ligation; ii) the vehicle group, which was injected with physiological saline $(0.1 \mathrm{ml} / 100 \mathrm{~g}$, i.p.) and underwent occlusion of the left coronary artery; iii) The OSR groups, which were subjected to the occlusion of the left coronary artery and treated with OSR at concentrations of $62.5,125$ or $250 \mathrm{mg} / \mathrm{kg}$. Physiological saline or OSR were administered for seven consecutive days, then 30 min after the final treatment, rats underwent surgery.

Measurement of infarct size. Six hours after the occlusion of the coronary artery, the hearts were swiftly excised and the left ventricles sectioned into transverse slices (2-mm thick) from the apex to the atrioventricular groove. The slices were then incubated in $1 \%$ triphenyltetrazolium chloride (TTC; Sigma-Aldrich, St. Louis, MO, USA) solution at $37^{\circ} \mathrm{C}$ for $30 \mathrm{~min}$. Images were captured with a digital camera (Canon 500D; Canon, Inc., Tokyo, Japan) and weighed. Normal myocardium was stained brick red, while areas without color indicated the ischemic heart muscle. The volume and weight as a percentage of the left ventricle were used to calculate the size of the infarcted area.

Determination of cardiac marker enzymes. Six hours after occlusion of the coronary artery, blood samples were collected in order to determine myocardium-specific enzymes, including creatine kinase (CK), MB isoenzyme of creatine kinase (CK-MB), lactate dehydrogenase (LDH) and cardiac troponin $\mathrm{T}$ (cTnT). The colorimetric method was employed to analyze the activity levels of CK, CK-MB and LDH according to the manufacturer's instructions (Nanjing Jiancheng Bioengineering Institute, Nanjing, China). Serum levels of cTnT were measured using a Diagnostics Elecsys 2010 Immunoassay system (Roche Diagnostics, Basel, Switzerland).

Measurement of malondialdehyde (MDA), superoxide dismutase (SOD), glutathione (GSH) and glutathione peroxidase (GSH-PX) activity. The level of MDA and the enzymatic activity of catalase (CAT), Cu/Zn-SOD, Mn-SOD and GSH-PX together with the non-enzymatic GSH were measured in the heart homogenate using the GSH-PX assay kit (colorimetric method) according to the manufacturer's instructions of this commercial assay kits (Nanjing Jiancheng Bioengineering Institute).

Measurement of NF- $\kappa B$ p 65 unit, TNF- $\alpha, I L-1 \beta, I L-6$ and IL-10 levels. The p65 subunit may be positively correlated with the activation of the NF- $\mathrm{BB}$ pathway; thus, the levels of NF- $\kappa B$ p65 were measured (Cayman Chemical, Ann Arbor, MI, USA), in addition to the levels of TNF- $\alpha$, IL-1 $\beta$, IL- 6 and IL-10, with commercial immunoassay kits (KCB7226, RTA00, RLB00, R6000B and R1000, respectively; R\&D Systems, Inc., Minneapolis, MN, USA) according to the manufacturer's instructions.

Assay of caspase-3 activity. The levels of the cleavage product of the chromogenic caspase substrate, Ac-DEVD-pNA (acetyl-Asp-Glu-Val-Asp p-nitroanilide), were measured to quantify the activity of caspase-3. The quantity of caspase-3 was measured using the colorimetric approach with the Caspase-3 Activity Assay Kit (Beyotime Institute of Biotechnology, Haimen, China). The protein samples from the heart were obtained by centrifugation at 13,500 $\mathrm{x}$ g for $20 \mathrm{~min}$. Approximately $50 \mu \mathrm{g}$ protein was added to a reaction buffer containing Ac-DEVD- $p \mathrm{NA}(2 \mathrm{mM})$, incubated at $37^{\circ} \mathrm{C}$ for $4 \mathrm{~h}$, and the absorbance of yellow $p$ NA (the cleavage product) was calculated with a spectrometer (Shanghai Chenguang Medical 
Technologies Co., Ltd., Shanghai, China) at a wavelength of $405 \mathrm{~nm}$. The specific activity of caspase-3, which was normalized to the total protein in the heart tissue, was then expressed as fold of the baseline caspase- 3 activity of the control group.

Statistical analysis. Data were expressed as the mean \pm standard deviation, with $n=6$ in each group. Experimental results were analyzed using one-way analysis of variance, followed by Dunnett's test for individual comparisons between each group. All statistical analyses were conducted using SPSS software, version 13.0 (SPSS, Inc., Chicago, IL, USA). $\mathrm{P}<0.05$ was considered to represent a statistically significant difference.

\section{Results}

OSR treatment reduces myocardial infarct size in an AMI rat model. The chemical structure of OSR is presented in Fig. 1. The infarction size in the infarcted group was $41.66 \pm 2.56 \%$. Subsequent to treatment with OSR at a dose of $62.5,125$ and $250 \mathrm{mg} / \mathrm{kg}$, the infarcted area was significantly reduced to $35.43 \pm 3.54 \%, 30.68 \pm 3.42 \%$ and $28.59 \pm 2.98 \%$, respectively $(\mathrm{P}<0.01$ vs. the infarcted group; Fig. 2).

$O S R$ reduces activity of $C K, C K-M B$ and $L D H$, and the level of cTnT in an AMI rat model. The measured levels of serum CK, CK-MB, LDH and cTnT are shown in Fig. 3. An increase in the levels of all the measured proteins $(\mathrm{P}<0.01)$ was observed in infarcted rats compared with the sham controls. Treatment with OSR reduced the levels of the proteins in a dose-dependent manner, and all were significantly reduced compared with the vehicle-treated group $(\mathrm{P}<0.01)$.

Effects of OSR treatment on the content of MDA and activity levels of CAT, Cu/Zn-SOD, Mn-SOD, GSH and GSH-PX in a rat model of AMI. To explore whether OSR exerted cardioprotective effects against AMI via an antioxidant mechanism, the level of MDA and the activity levels of CAT, $\mathrm{Cu} / \mathrm{Zn}-\mathrm{SOD}$, Mn-SOD, GSH and GSH-PX in heart homogenate were determined, as illustrated in Fig. 4. A marked increase was observed in the level of MDA (an index of lipid peroxidation) in infarcted rats $(\mathrm{P}<0.01$ vs. sham operation group). Pretreatment with OSR at different doses $(62.5,125$ and $250 \mathrm{mg} / \mathrm{kg})$ significantly diminished the infarction-mediated lipid peroxidation $(\mathrm{P}<0.01$ vs. the vehicle group) in a dose-dependent manner. The activity levels of antioxidants and anti-oxidative enzymes were also analyzed in the heart homogenate of control and experimental groups. Rats with AMI exhibited significant reductions in levels of CAT, $\mathrm{Cu} / \mathrm{Zn}-\mathrm{SOD}, \mathrm{Mn}-\mathrm{SOD}, \mathrm{GSH}$ and GSH-PX ( $<<0.01$ vs. the sham-operated rats). OSR treatment significantly augmented levels of CAT, Cu/Zn-SOD, Mn-SOD, GSH and GSH-PX in the hearts of infarcted rats compared with levels in the vehicle group.

OSR reduces activity levels of $N F-\kappa B$ p65, TNF- $\alpha, I L-1 \beta$, $I L-6$ and $I L-10$ in a rat model of AMI. Fig. 5 displays the effects of OSR on molecules involved in the inflammatory response (including $\mathrm{NF}-\kappa \mathrm{B}$ p 65 , TNF- $\alpha$, IL-1 $\beta$, IL- 6 and IL-10) in a rat model of AMI. It was noted that the levels of NF- $\kappa$ B p65, TNF- $\alpha$, IL-1 $\beta$, IL-6 and IL-10 $(\mathrm{P}<0.01)$ were

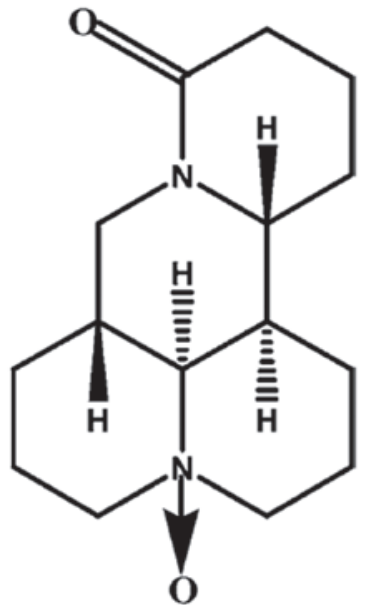

Figure 1. Chemical structure of oxysophoridine.

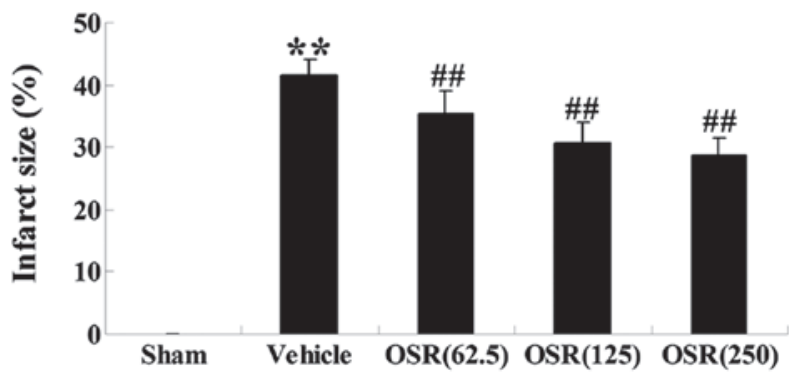

Figure 2. Effects of OSR on infarct size of hearts in a rat model of acute myocardial infarction (the mean \pm standard deviation, $n=6$ ). Groups were as follows: Sham, sham-operated; vehicle, vehicle-treated; OSR, OSR-treated (dose, $\mathrm{mg} / \mathrm{kg}$ ). ${ }^{* *} \mathrm{P}<0.01$ vs. the sham group, ${ }^{\# \#} \mathrm{P}<0.01$ vs. the vehicle-treated group. OSR, oxysophoridine.

significantly increased in the vehicle-treated myocardial infarction group. Treatment with OSR led to clear reductions in levels of NF- $\kappa$ B p65, TNF- $\alpha$, IL-1 $\beta$, IL-6 and IL-10 $(\mathrm{P}<0.01)$ in a dose-dependent manner, compared with the vehicle-treated group.

Effects of OSR on the caspase-3 activity in a rat model of $A M I$. In order to determine whether OSR could attenuate the apoptotic damage induced by myocardial infarction, the activity level of caspase-3, an executioner molecule in the apoptotic signaling pathway, was determined by colorimetric analysis. As presented in Fig. 6, caspase-3 activity in the vehicle group was significantly enhanced $(\mathrm{P}<0.01)$ compared with the sham group. In the OSR pretreatment $(62.5,125$ and $250 \mathrm{mg} / \mathrm{kg}$ ) groups, there was a significant reduction in caspase-3 activity $(\mathrm{P}<0.01)$ compared with that of the vehicle group.

\section{Discussion}

AMI is a predominant ischemic disease, for which TTC staining and enhanced cardiac magnetic resonance imaging can be used to delineate the acute necrotic infarct zone directly. The major findings of the present study in AMI rat models clarified that: (i) OSR was able to diminish the myocardial infarct size and the activity levels of serum CK, CK-MB and 
A

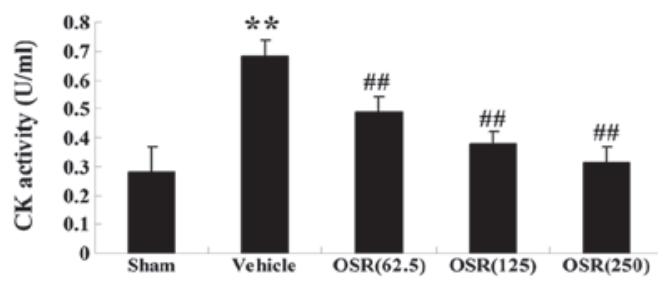

C

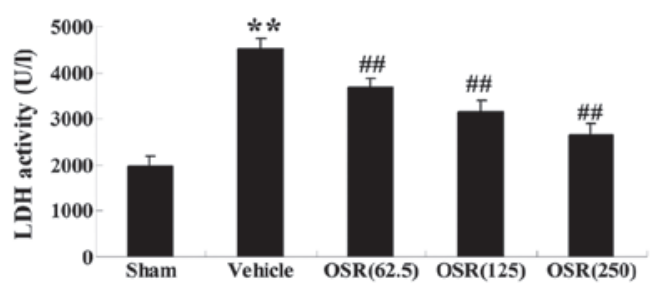

B

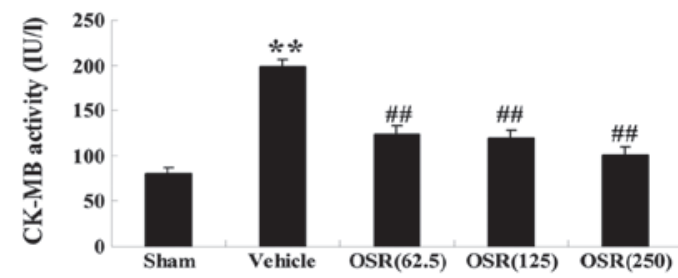

D

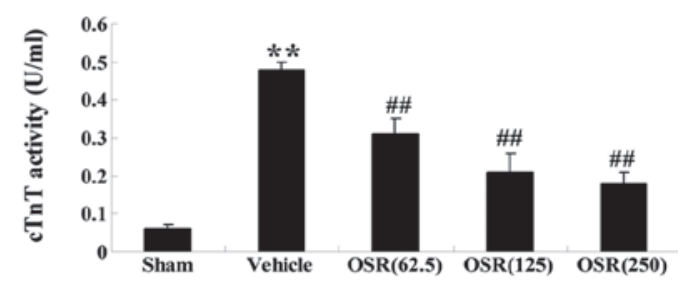

Figure 3. Effects of OSR on serum (A) CK, (B) CK-MB and (C) LDH activities together with (D) cTnT level in a rat model of acute myocardial infarction (the mean \pm standard deviation, $\mathrm{n}=6$ ). Groups were as follows: Sham, sham-operated; Vehicle, vehicle-treated; OSR, OSR-treated (dose, $\mathrm{mg} / \mathrm{kg})$. As cTnT serum level positively correlates with $c \mathrm{TnT}$ activity, cTnT serum can directly reflect $\mathrm{cTnT}$ activity. ${ }^{* *} \mathrm{P}<0.01$ vs. the sham group, ${ }^{\# \#} \mathrm{P}<0.01$ vs. the vehicle group. OSR, oxysophoridine; CK, creatine kinase; CK-MB, MB isoenzyme of CK; LDH, lactate dehydrogenase; cTnT, cardiac troponin T.

A

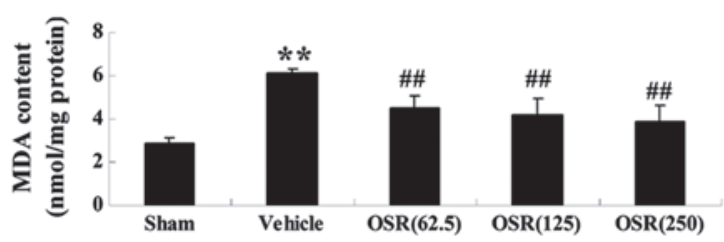

C

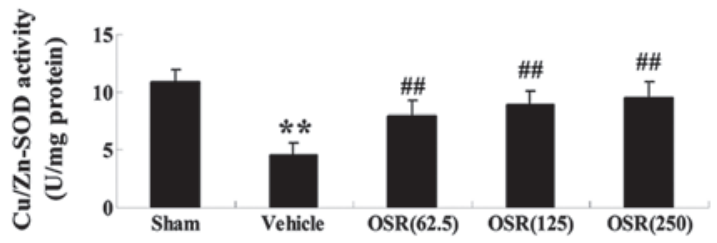

$\mathbf{E}$

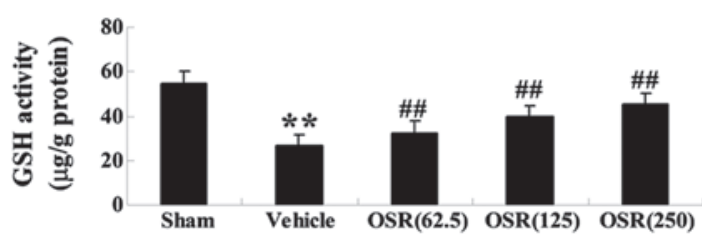

B

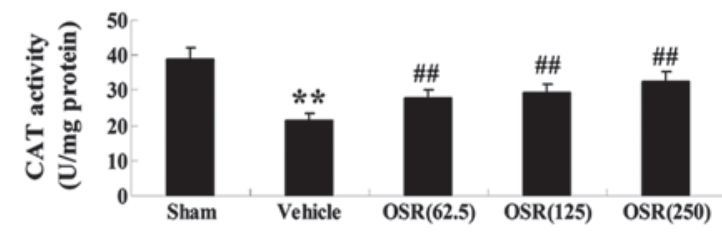

D

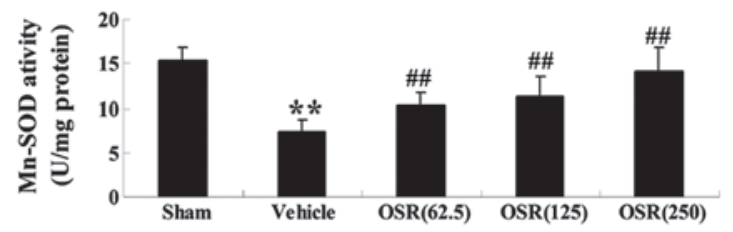

$\mathbf{F}$

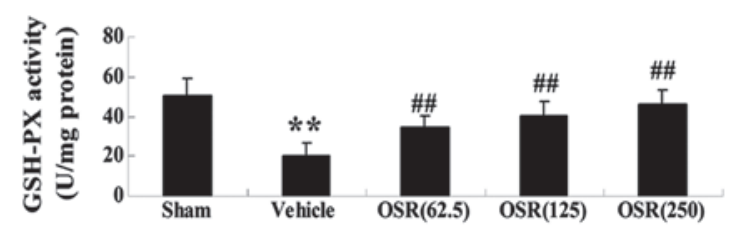

Figure 4. Effects of OSR on the content of (A) MDA and activities of (B) CAT, (C) Cu/Zn-SOD, (D) Mn-SOD, (E) GSH and (F) GSH-PX in a rat model of acute myocardial infarction (the mean \pm standard deviation, $n=6$ ). Groups were as follows: Sham, sham-operated; Vehicle, vehicle-treated; OSR, OSR-treated (dose, $\mathrm{mg} / \mathrm{kg}$ ). ${ }^{* *} \mathrm{P}<0.01$ vs. the sham group, ${ }^{\# \#} \mathrm{P}<0.01$ vs. the vehicle group. OSR, oxysophoridine; MDA, malondialdehyde; CAT, catalase; Cu/Zn-SOD, copper/zinc-superoxide dismutase; Mn-SOD, manganese-SOD; GSH, glutathione; GSH-PX, GSH-peroxidase.

LDH together with levels of cTnT protein; (ii) OSR significantly suppressed lipid peroxidation (quantified by the level of MDA production) but increased endogenous antioxidant enzymes (CAT, Cu/Zn-SOD, Mn-SOD and GSH) in addition to the non-enzymatic scavenger (GSH-PX); (iii) OSR inhibited the activities of a number of major inflammatory factors, including NF- $\kappa$ B p 65 , TNF- $\alpha$, IL-1 $\beta$, IL-6 and IL-10; and (iv) OSR treatment resulted in reduced activity of caspase-3. These findings support the hypothesis that OSR exerts a cardioprotective effect against injury resulting from AMI, through anti-oxidative, anti-inflammatory and anti-apoptotic mechanisms.

OSR, which is derived from the natural alkaloid, Sophora alopecuroides $L$. has been indicated to produce various pharmacological effects. It has been previously reported that OSR was able to ameliorate focal brain ischemia 
A

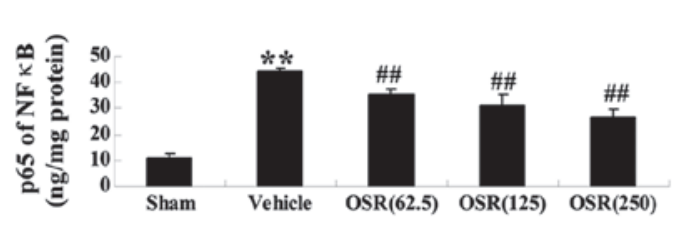

C

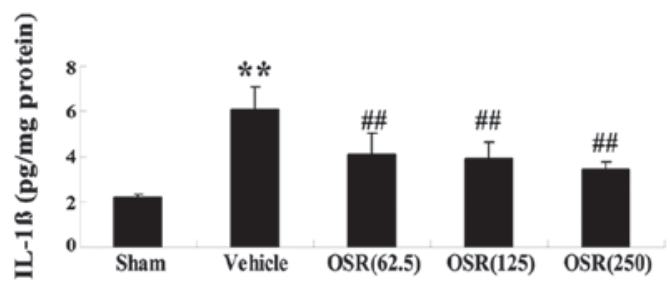

B

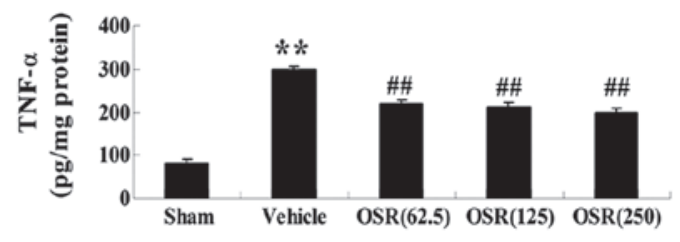

D

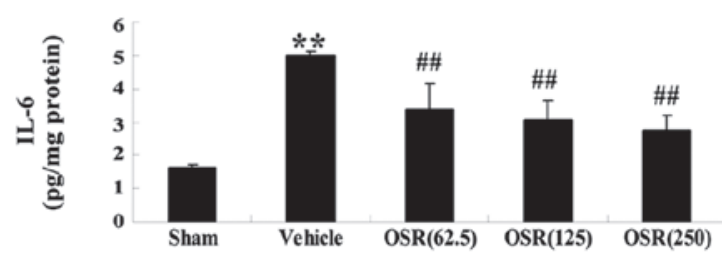

$\mathbf{E}$

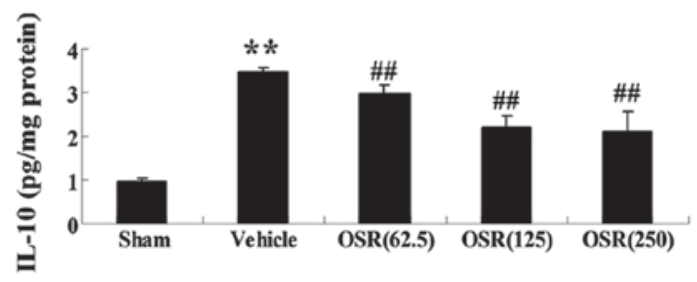

Figure 5. Effects of OSR on the activities of (A) NF $\kappa B$ p65, (B) TNF- $\alpha$, (C) IL-1 $\beta$, (D) IL-6 and (E) IL-10 in a rat model of acute myocardial infarction (the mean \pm standard deviation, $\mathrm{n}=6$ ). Groups were as follows: Sham, sham-operated; Vehicle, vehicle-treated; OSR, OSR-treated $(\mathrm{dose}, \mathrm{mg} / \mathrm{kg}) .{ }^{* *} \mathrm{P}<0.01 \mathrm{vs}$. the sham group, ${ }^{\# \#} \mathrm{P}<0.01$ vs. the vehicle group. OSR, oxysophoridine; NF, nuclear factor; TNF, tumor necrosis factor; IL, interleukin.

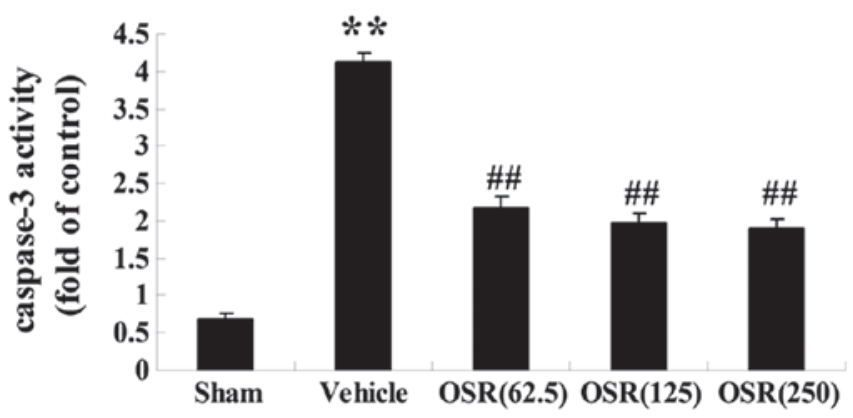

Figure 6. Effects of OSR on the caspase-3 activity in a rat model of acute myocardial infarction (the mean \pm standard deviation, $n=6$ ). Groups were as follows: Sham, sham-operated; Vehicle, vehicle-treated; OSR, OSR-treated (dose, $\mathrm{mg} / \mathrm{kg}$ ).* $\mathrm{P}<0.01$ vs. the sham group, ${ }^{\# \#} \mathrm{P}<0.01$ vs. the vehicle group. OSR, oxysophoridine.

in mice via inhibiting oxidative stress and apoptosis (9). The present study extended the therapeutic profile of OSR and disclosed a potent cardioprotective role against AMI in rats.

The infarct size, and levels of myocardium-specific enzymes, including CK, CK-MB, LDH and cTnT are regarded as key factors for the evaluation of cardiac injury resulting from ischemic heart disease. Significant elevations in infarct size and the activity of these myocardium-specific enzymes in rats were identified during $\operatorname{AMI}(6,11)$. In addition, cTnT, a specific and sensitive indicator of ischemic insults, was reported to be produced when myocardial necrosis occurs (12). Consistent with these previous studies, the present study revealed that the infarct size and the activity levels of CK, CK-MB, LDH and cTnT were significantly elevated in infarcted rats. Furthermore, these indices were all reduced following OSR treatment in AMI-induced rats, demonstrating the cardioprotective effect of OSR.

Excessive production of oxygen free radicals is involved in the generation of ischemic impairments. Under normal physiological conditions, the generation of oxygen free radicals is controlled by endogenous antioxidant enzymes, such as CAT, $\mathrm{Cu} / \mathrm{Zn}-\mathrm{SOD}, \mathrm{Mn}-\mathrm{SOD}$, GSH-PX and low-molecular weight antioxidants, such as non-enzymic GSH. Treatment with antioxidants and radical scavengers is beneficial for ischemic stroke therapy $(13,14)$. In addition, MDA serves as a sensitive index for evaluating lipid peroxidation, as it is composed of products of the decomposition of fatty acids of myocardial membranes. Results from the current study demonstrated that OSR significantly diminished the content of MDA and increased the activity of $\mathrm{Cu} / \mathrm{Zn}-\mathrm{SOD}, \mathrm{Mn}-\mathrm{SOD}, \mathrm{GSH}$ and GSH-PX in the infarcted rats. This implies that the cardioprotection of OSR against AMI in rats was associated with its anti-oxidative properties.

The inflammatory response also participates in the pathogenesis of ischemic heart diseases $(15,16)$. It was reported that the activation of $N F-\kappa B$ led to the marked release of proinflammatory mediators, such as TNF- $\alpha$ and IL-1 $\beta$. In fact, inhibition of the $\mathrm{NF}-\kappa \mathrm{B}$ pathway may improve adverse left ventricular remodeling and cardiac dysfunction during myocardial infarction (17). The present study demonstrated that OSR suppressed the increase of NF- $\mathrm{B}$ p65 and reduced the activity levels of TNF- $\alpha$, IL-1 $\beta$, IL- 6 and IL-10 in rats subjected to myocardial infarction. Ruan et al (18) noted that OSR exerted anti-inflammatory effects against D-galactose-treated rats (18), which is in agreement with the findings of the current study. OSR was also demonstrated to inhibit nuclear translocation of $N F-\kappa B$ and reduce the 
increased levels of proinflammatory factors during transient focal cerebral ischemia (19). These findings suggest that OSR exerts cardioprotective effects subsequent to AMI through its anti-inflammatory profile.

Oxidative damage may facilitate mitochondrial dysfunction and subsequently activate an apoptotic cascade following cardiac impairments. To further explore the amelioration of the cardiac injuries resulting from AMI subsequent to OSR treatment, the activity of apoptosis-related proteins was assessed in the hearts of the rats. As evolutionarily conserved cysteinyl proteases, caspases serve a crucial function in cellular apoptosis, and caspase- 3 is a critical molecule in the caspase-dependent apoptotic cascade. It has been previously reported that caspase-3 activates diverse substrates that lead to DNA fragmentation and cell death (20), and upregulation of caspase-3 was observed following AMI $(6,10)$. In the present study, OSR significantly reduced caspase- 3 activity in infarcted rats. Consistent with these results, Wang and Tang (7) indicated that OSR significantly reduced the activity of caspase- 3 in Alzheimer's disease. Furthermore, the overexpression of caspase-3 was also suppressed in mice subjected to focal cerebral ischemic injury (9). The present investigation revealed a reduced level of caspase-3 activity in the AMI-induced rats treated with OSR, suggesting that its cardioprotective ability was acting through its anti-apoptotic profile.

The results of the current study demonstrated that OSR is able to attenuate the AMI-induced injury in rats and that this cardioprotection may be linked with its anti-oxidative, anti-inflammatory and anti-apoptotic properties. These findings imply that OSR has potential as a novel cardioprotective agent for treating AMI, but further studies are required in order to verify the results.

\section{References}

1. Yang B, Lin H, Xiao J, et al: The muscle-specific microRNA miR-1 regulates cardiac arrhythmogenic potential by targeting GJA1 and KCNJ2. Nat Med 13: 486-491, 2007.

2. Chan PH: Mitochondria and neuronal death/survival signaling pathways in cerebral ischemia. Neurochem Res 29: 1943-1949, 2004.

3. Müller DN, Mervaala EM, Dechend R, et al: Angiotensin II (AT(1)) receptor blockade reduces vascular tissue factor in angiotensin II-induced cardiac vasculopathy. Am J Pathol 157: 111-122, 2000.

4. Speir E: Cytomegalovirus gene regulation by reactive oxygen species. Agents in atherosclerosis. Ann NY Acad Sci 899 363-374, 2000.
5. Tanaka M, Mokhtari GK, Terry RD, et al: Overexpression of human copper/zinc superoxide dismutase (SOD1) suppresses ischemia-reperfusion injury and subsequent development of graft coronary artery disease in murine cardiac grafts. Circulation 110 (Suppl 1): II200-II206, 2004.

6. Guo J, Li HZ, Wang LC, et al: Increased expression of calcium-sensing receptors in atherosclerosis confers hypersensitivity to acute myocardial infarction in rats. Mol Cell Biochem 366: 345-354, 2012.

7. Wang R and Tang XC: Neuroprotective effects of huperzine A. A natural cholinesterase inhibitor for the treatment of Alzheimer's disease. Neurosignals 14: 71-82, 2005.

8. Yu W, Liu Q and Zhu S: Carvacrol protects against acute myocardial infarction of rats via anti-oxidative and anti-apoptotic pathways. Biol Pharm Bull 36: 579-584, 2013.

9. Wang TF, Lei Z, Li YX, et al: Oxysophoridine protects against focal cerebral ischemic injury by inhibiting oxidative stress and apoptosis in mice. Neurochem Res 38: 2408-2417, 2013.

10. Hong-Li S, Lei L, Lei S, et al: Cardioprotective effects and underlying mechanisms of oxymatrine against ischemic myocardial injuries of rats. Phytother Res 22: 985-989, 2008.

11. Ming X, Tongshen W, Delin W and Ronghua Z: Cardioprotective effect of the compound yangshen granule in rat models with acute myocardial infarction. Evid Based Complement Alternat Med 2012: 717123, 2012.

12. Katus HA, Remppis A, Scheffold T, Diederich KW and Kuebler W: Intracellular compartmentation of cardiac troponin T and its release kinetics in patients with reperfused and nonreperfused myocardial infarction. Am J Cardiol 67: 1360-1367, 1991.

13. Yamada J, Yoshimura S, Yamakawa H, et al: Cell permeable ROS scavengers, Tiron and Tempol, rescue PC12 cell death caused by pyrogallol or hypoxia/reoxygenation. Neurosci Res 45: 1-8, 2003.

14. Li Y, Bao Y, Jiang B, et al: Catalpol protects primary cultured astrocytes from in vitro ischemia-induced damage. Int $\mathrm{J}$ Dev Neurosci 26: 309-317, 2008.

15. Frantz S, Fraccarollo D, Wagner H, et al: Sustained activation of nuclear factor kappa B and activator protein 1 in chronic heart failure. Cardiovasc Res 57: 749-756, 2003.

16. Wong SC, Fukuchi M, Melnyk P, Rodger I and Giaid A: Induction of cyclooxygenase-2 and activation of nuclear factor-kappaB in myocardium of patients with congestive heart failure. Circulation 98: 100-103, 1998.

17. Yoshiyama M, Omura T, Takeuchi K, et al: Angiotensin blockade inhibits increased JNKs, AP-1 and NF- kappa B DNA-binding activities in myocardial infarcted rats. J Mol Cell Cardiol 33: 799-810, 2001

18. Ruan Q, Liu F, Gao Z, et al: The anti-inflamm-aging and hepatoprotective effects of huperzine A in D-galactose-treated rats. Mech Ageing Dev 134: 89-97, 2013.

19. Wang ZF, Wang J, Zhang HY and Tang XC: Huperzine A exhibits anti-inflammatory and neuroprotective effects in a rat model of transient focal cerebral ischemia. J Neurochem 106: 1594-1603, 2008.

20. Manabat C, Han BH, Wendland M, et al: Reperfusion differentially induces caspase-3 activation in ischemic core and penumbra after stroke in immature brain. Stroke 34: 207-213, 2003. 